\title{
"Volver a la tierra": dimensiones territoriales del trabajo como delimitantes de las opciones laborales para las mujeres en Madrid, Cundinamarca*
}

DOI: https://doi.org/10.18046/recs.iEspecial.3250

"Back to soil": Territorial Dimensions of Work as Delimiters of Labor
Options for Women in Madrid Municipality (Colombia)

María Carolina Olarte-Olarte**

Universidad de los Andes (Bogotá, Colombia)

Guisella Lara-Veloza ${ }^{* * *}$

Universidad del País Vasco (Bilbao, España)

\footnotetext{
${ }^{*}$ Este artículo forma parte de una investigación más amplia sobre movimientos sociales y comunitarios, desarrollada en conjunto con la profesora Juliana Flórez-Flórez del Instituto Pensar, de la Pontificia Universidad Javeriana de Bogotá. El artículo contó con financiación del Fondo de Apoyo para Profesores Asistentes de la Universidad de Los Andes. Agradecemos a Ángela María Sánchez por toda su colaboración como asistente de investigación. Artículo de investigación recibido el 13.11.2018 y aceptado el 03.05.2019.

${ }^{* *}$ Profesora asistente de la Facultad de Derecho de la Universidad de los Andes (Colombia). Correo electrónico:mc.olarteo@uniandes.edu.co ORCID: https://orcid.org/oooo-0oo2-6510-3815

*** Estudiante de la Maestría en Desarrollo y Cooperación Internacional del Instituto Hegoa de la Universidad del País Vasco (España).Correo electrónico: guisellalveloza@gmail.com
} 


\section{Cómo citar/How to cite}

Olarte-Olarte, María Carolina; Lara-Veloza, Guisella (2019). "Volver a la tierra": dimensiones territoriales del trabajo como delimitantes de las opciones laborales para las mujeres en Madrid, Cundinamarca. Revista CS, núm. especial, 167-198. https://doi.org/10.18046/recs.iEspecial.3250 
Resumen

Abstract

Este artículo propone considerar las dimensiones territoriales del trabajo desde una perspectiva de género y como elemento de análisis para la academia del derecho laboral. A través de un estudio de caso de la asociación Asoquimad, en Madrid, Cundinamarca, el artículo identifica una serie de determinantes territoriales que subyacen a las trayectorias laborales de mujeres que buscan, en escenarios periurbanos, hacer un tránsito del trabajo en la agroindustria floricultora al trabajo cooperativo en economías agroecológicas y solidarias de pequeña escala. El caso ilustra cómo dinámicas globales, regionales y locales se intersectan en la delimitación de las opciones laborales de estas mujeres en un contexto de transformación y disputa de lo rural. La lectura de las limitantes enfrentadas por las mujeres posibilita una comprensión más profunda del trabajo, así como de sus articulaciones específicas con las transformaciones periurbanas.

\section{PALABRAS CLAVE:}

perspectiva de género, constitución laboral, transformaciones periurbanas

This article proposes to consider the territorial dimensions of work from a gender perspective and as an element for analysis in labor law academy. Through a case study of the Asoquimad association in Madrid, Cundinamarca, the article identifies a series of territorial determinants that underlie the employment history of women who seek, in peri-urban scenarios, to transit from working in the floriculture agroindustry to cooperative work in small-scale agroecological and solidarity economies. The case illustrates how global, regional, and local dynamics intersect in the delimitation of the labor options these women have access to in a context of transformation and dispute of what "the rural" is. The reading of the constraints faced by women enables a deeper understanding of labor as well as of its specific articulations with peri-urban transformations.

\section{KEYWORDS:}

Gender Perspective, Labor Constitution, Peri-urban Transformations 



\section{Introducción}

“(...) Mimamá siempre buscaba un pedacito detierra donde sembrar un par de papas y arracacha para luego hacer una comida y compartir con los otros trabajadores. Constantemente, defendía la pequeña área sembrada de los supervisores. Varias mujeres también siembran jardincitos en los rincones de los invernaderos. Es una manera de relacionarse de forma diferente en ese ambiente. Sabemos que algunas personas incluso enterraban aguardiente debajo de la tierra, sobre todo en diciembre donde "entonarse" resultaba crucial para sobrevivir las largas jornadas".

Lucrecia (pseudónimo), comunicación personal, $12.2 \mathrm{O} 14^{1}$

Desde 2009 y por cerca de siete años, Asoquimad (Asociación de Quinua de Madrid), una empresa asociativa de trabajo para la producción de quinua, hortalizas, aromáticas y la recuperación de semillas ancestrales de la región, creada por un grupo de mujeres en Madrid, Cundinamarca, usó un terreno de la alcaldía del municipio y lo transformó en una huerta orgánica de la que obtenía un sustento. Esta explotación hacía parte del proyecto de huertas orgánicas promovido por la Red de Mujeres Populares de la Sabana, así como de las políticas de lugar² adelantadas por la Escuela de Mujeres de Madrid (EMM) y la Asociación Herrera. La organización de huertas orgánicas representa una opción laboral y de vida distinta a la agroindustria de flores, el mayor referente laboral en el municipio en las últimas cuatro décadas. Se trataba de una apuesta política, territorial y laboral de las mujeres de Asoquimad por convertirse en trabajadoras cooperativas a través de la producción de alimentos en esquemas solidarios de pequeña escala. La huerta se convirtió en fuente de sustento alimentario, pero también en el eje de un proyecto de educación popular para el trabajo digno, la apropiación del territorio y el cuidado de sus cuerpos afectados por la floricultura.

1. Todas las citas en este artículo referidas como "comunicación personal" fueron obtenidas mediante entrevistas semiestructuradas, entrevistas grupales y entrevistas informales, entre otras técnicas y prácticas. La denominación "comunicación personal” responde a las normas editoriales de la revista.

2. La noción de políticas de lugar es una forma de nombrar prácticas que, desde la experiencia de los movimientos sociales, muestran apuestas políticas y epistemológicas locales. Son prácticas ancladas a un lugar, que suceden de manera particular, pero en diálogo con lo global, y que dan lugar a dinámicas sociales construidas desde abajo, generando procesos sociales propios mediante los cuales se analiza la realidad y se busca transformarla. Para una aproximación más detallada, ver Harcourt (2017). 
En 2015, la alcaldía le pidió a Asoquimad la devolución del terreno, para lo cual adujo diversas razones relacionadas con la productividad del mismo y el cumplimiento de objetivos del plan de desarrollo municipal. En este proceso, la denominación del terreno como "Granja Demostrativa" y las interacciones con diferentes funcionarios locales permitían entrever unas lecturas institucionales sobre su uso y destinación, que oscilaban entre invocaciones al desarrollo y una promoción elusiva del campo. Las trayectorias laborales de las mujeres, su saber agroecológico y el trabajo invertido en el terreno no fueron parte de esas lecturas. Una vez abandonaron la huerta, el terreno se convirtió en potrero hasta que, en noviembre de 2017 , se ejecutó un contrato de mantenimiento cuya duración, no obstante, fue de solo quince días. La pérdida del terreno significó un quiebre crítico de la apuesta política, laboral y territorial de "volver a la tierra", relacionada con el cultivo de alimentos como una opción laboral alterna a la oferta predominante en un área periurbana sometida a rápidas y constantes transformaciones. Esta pérdida es también un mensaje a los movimientos de la Sabana, principalmente en la Sabana Occidente, que debaten el significado del desarrollo, lo rural y el territorio.

¿Qué puede decirle la situación de las mujeres de Asoquimad al derecho y la enseñanza del derecho laboral? Las representaciones institucionales y corporativas del trabajo en la Sabana Occidente se centran en una perspectiva de mercado laboral que, con frecuencia, reducen el trabajo a un número de empleos formales e informales creados, y a indicadores porcentuales y estadísticos del caso. En contraste, la experiencia de estas mujeres es un ejemplo del reconocimiento del trabajo como una actividad social mediada por varias dimensiones sustantivas que sobrepasan las formas jurídicas o funciones económicas del derecho laboral.

Es así como, cuando las mujeres de Asoquimad y de la EMM hablan del "trabajo" se refieren a una constelación de actividades y vivencias que no pueden desarticularse de sus trayectorias laborales pasadas, de las transformaciones del territorio en la Sabana en general, de su cotidianeidad, de la historia que cuentan sus cuerpos ni de sus apuestas políticas, que han significado hacer una transición a un sistema más cooperativo. Como señalaremos más adelante, no es posible desligar el presente de su trabajo pasado en la industria de flores, del cultivo y la producción de alimentos en el terreno perdido-y ahora en los patios caseros-, del trabajo reproductivo no pago en sus hogares e, incluso, del tiempo dedicado a la movilización social por la defensa del territorio y el agua que, para muchas de ellas, es "un tercer trabajo" (mujer miembro de Asoquimad, comunicación personal, 04.2015). Estas actividades son todas manifestaciones del trabajo que define la realidad de las mujeres de Asoquimad.

En este artículo, argumentamos que una lectura situada y relacional del trabajo por parte de Asoquimad, y de la EMM en general, interpela a la construcción de lo 
laboral en el derecho. Exige pensar lo laboral siempre en relación con otras categorías como el territorio, las trayectorias laborales y la historia de los cuerpos. En este orden, la disputa por el terreno no solo permite construir la idea de lo laboral en los cuerpos y territorios de la Sabana Occidente, sino visibilizar de qué manera esa construcción responde además a presiones que exceden lo local. En particular, esperamos cuestionar la centralidad que los datos de empleabilidad tienen en las representaciones institucionales y corporativas del trabajo - del de las mujeres, en particular- en un área periurbana como la Sabana Occidente de Bogotá. En últimas, buscamos retar esta mirada reduccionista del trabajo en el derecho laboral, con el fin de propiciar una revisión futura de cómo se relaciona con indicadores estadísticos y porcentuales.

La primera parte ofrece algunas precisiones político-metodológicas que motivan y enmarcan la identificación en las secciones subsiguientes sobre las relaciones entre trabajo y territorio propuestas desde la experiencia de Asoquimad. Con esta finalidad, la metodología está construida sobre la articulación de la invitación a cuestionar qué se entiende por trabajo, en general, y en el derecho, en particular, dentro del marco de las discusiones surgidas en el encuentro que dio lugar a este número especial de la Revista CS, y la apuesta por la coproducción de conocimiento con los movimientos sociales desde una perspectiva feminista de la investigación sobre la vivencia y comprensión del territorio por parte de Asoquimad.

La segunda parte del texto aborda, en más detalle, las motivaciones y contexto de Asoquimad. En este marco, relatamos la intrincada serie de respuestas institucionales ofrecidas por el gobierno local de Madrid para justificar la expulsión del terreno que usaron las mujeres de la asociación por casi siete años en desarrollo de un proyecto laboral, territorial y político para la producción de alimentos. Este proyecto -consideran- les permitió construir una alternativa autónoma de trabajo respetuosa de sus cuerpos y de su tiempo, luego de haber salido del sistema laboral agroindustrial. Aquí resaltamos, a manera introductoria, cómo la disputa por este terreno es una manifestación tanto de una discusión más amplia por el desarrollo en la Sabana de Bogotá, ligada a presiones locales, regionales y globales, como de los obstáculos institucionales locales para la producción solidaria y en pequeña escala de alimentos. Este contexto permitirá contrastar las opciones laborales de las mujeres que hicieron parte de Asoquimad con las lecturas institucionales de su trabajo en la huerta.

La tercera parte afirma la centralidad del carácter periurbano del municipio con relación a las posibilidades y límites de iniciativas dirigidas a la producción de alimentos en economías solidarias y de pequeña escala. Sugerimos que las reducidas opciones laborales de las mujeres de Asoquimad pueden ser entendidas como una manifestación de la dimensión territorial del trabajo, en la que sus opciones labo- 
rales están profundamente conectadas con las opciones y procesos de organización e intervención territorial.

En el marco periurbano, la última parte articula la contribución central del texto. Proponemos que la problematización de la idea de trabajo debe ir de la mano con una dimensión territorial que, en este caso, se enmarca en el contexto del desarrollo periurbano que reproduce dinámicas de comercio global. Con este fin, la sección identifica las delimitantes territoriales de las opciones laborales -pero también políticas- de mujeres que decidieron optar por un modo de vida fuera de la industria de la floricultura y de otras alternativas (como el bodegaje), que responden a transformaciones territoriales que excluyen o, al menos, dificultan significativamente la posibilidad de producir alimentos como forma de subsistencia y proyecto de vida.

Como conclusión, proponemos que el análisis de las dimensiones territoriales del trabajo posibilita una comprensión más profunda y situada del trabajo de las mujeres. En particular, el proyecto de Asoquimad y su disputa casi kafkiana por un espacio para cultivar pueden contribuir a desestabilizar lecturas institucionales y desarrollistas del trabajo de las mujeres que invisibilizan la producción de espacialidades para la permanencia campesina en un territorio que no es ni rural ni urbano.

\section{Precisiones político-metodológicas}

Este artículo es una apuesta metodológica y política que busca visibilizar la conexión entre las transformaciones del territorio y las opciones laborales de las mujeres, más allá de una lectura desde las cifras de creación de empleo. Así, cuestiona una difundida lectura del trabajo desde indicadores de empleo en Madrid y la Sabana Occidente, y propone una problematización de la idea de trabajo a partir de una dimensión territorial. Se trata de entender entonces de qué manera las apropiaciones y transformaciones del territorio no pueden desarticularse de una lectura en perspectiva crítica de las opciones laborales. Con este fin, la metodología se inspira en la invitación de Ruth Dukes $(2014 ; 2018)$ a cuestionar qué se entiende por trabajo, en general, y en el derecho, en particular, para posicionar una serie de determinantes territoriales de las opciones laborales, construidos a partir de la coproducción de conocimiento sobre la vivencia y comprensión del territorio por parte de Asoquimad, en un escenario periurbano donde intentan hacer un tránsito del trabajo en la agroindustria de las flores a trabajadoras cooperativas.

Con relación al cuestionamiento a la lectura predominante del trabajo, la representación corporativa e institucional del trabajo de las mujeres trabajadoras en Madrid ha reflejado una perspectiva de mercado laboral basada, primordialmente, 
en el número de empleos creados, que se concentran, ante todo, en reportes e informes sobre empleos formales directos e indirectos en la floricultura y la zona franca de Mosquera (Departamento Administrativo Nacional de Estadística, 2010; Superintendencia de Sociedades, 2016; Solano Mejía, 2016; Alcaldía de Madrid, 2014). Así, solo para citar un ejemplo, el Ministerio del Trabajo (2012) describió al sector floricultor en Madrid y a la zona franca de occidente -colindante con dicho municipio-, a partir del número de empleos que los posicionaban como las principales fuentes laborales del municipio. Para ello, indicó que, en 2012, el sector sumaba el $68 \%$ del empleo, al generar 18.0oo puestos de trabajo directos y 11.000 indirectos, mientras se estimaba que la zona franca de occidente generara por lo menos 15.000 empleos en la Sabana. La centralidad del número de empleos creados y esperados en estas representaciones es reflejo de una lectura difundida y reduccionista del mercado laboral, que está construida sobre indicadores de empleabilidad donde el número de empleados aparece como una objetividad sin historia ni lugar, y contribuye a intensificar lo que Christodoulidis (2018: 413) ha descrito como "la pérdida del lenguaje de la protección laboral y la solidaridad laboral", resultado de la extendida interpretación del derecho laboral como "regulación del mercado laboral y como 'gobernanza económica"'3.

En contraste con esta interpretación, proponemos una lectura del trabajo de las mujeres en Madrid que incluya unas dimensiones territoriales que permitan identificar y debatir lo que Ruth Dukes (2014: 6) ha descrito como "la naturaleza inherentemente política de las normas e instituciones laborales, así como de sus funciones económicas". Para ello, nos inspiramos en su noción de la constitución laboral y su más reciente invitación a construir una sociología económica del derecho laboral ${ }^{4}$. La idea de la constitución laboral es propuesta como un marco de análisis que dirige la atención hacia las consecuencias que, para las personas trabajadoras, implica "la reducción y desaparición de los espacios para la deliberación democrática y la toma de decisiones democráticas a medida que los mercados continúan expandiéndose" (Dukes, 2014: 221). Desde esta perspectiva, el principio de democracia económica resulta, entonces, central para el estudio -y enseñanza- del derecho laboral. Este principio resitúa las discusiones del derecho laboral frente a las preguntas que los

3. Traducción propia. En adelante, todas las citas del trabajo de Ruth Dukes son traducciones propias.

4. En su libro The Labour Constitution, Ruth Dukes (2014) busca contribuir a los debates sobre el alcance y significado del derecho laboral en un contexto donde complejos procesos de globalización retan las premisas sobre las que el campo originalmente fue construido y teorizado. El objeto más amplio de su libro es la defensa de una lectura de las normas laborales como "el producto de conflictos y acuerdos sobre su diseño y su interpretación o funcionamiento" (Dukes, 2014: 205). 
procesos de la globalización originan, como, por ejemplo, la necesidad de identificar no solo las diferencias entre diversas formas contemporáneas y tradicionales de trabajo, sino la manera en las que dichos procesos "han aislado las finanzas y comercio globales del control democrático" (Dukes, 2014: 196, 207).

En este marco de análisis, Dukes proyecta su propuesta para una sociología económica del derecho laboral $(2014 ; 2018)$. Se trata de un marco de análisis alternativo al mercado laboral, y a la eficiencia del mercado, para el estudio del derecho laboral $(2014)^{5}$. Como tal, permite, por ejemplo, evitar la descripción y aceptación de los mercados en términos abstractos y apolíticos, en los que el mercado laboral es identificado con los sitios "en los que compradores y vendedores de trabajo se encuentran" (2018: 420); dar visibilidad a conflictos de interés que van mucho más allá de la cuestión del precio; problematizar la idea de que el trabajo es algo que tiene que abordarse como una mercancía que se vende y se compra; y hacer de los mercados laborales un objeto de estudio, antes que el marco de análisis para las normas laborales (2018). Sobre este último punto, Dukes resalta la importancia de identificar los cambios en los contextos donde los contratos se forman, y, en particular, la centralidad que han adquirido las prácticas -intensamente profesionalizadas- de la gestión de recursos humanos y de sus asesores jurídicos (2018). La reorientación de la política pública de empleo y de las relaciones laborales en múltiples países ha sido uno de estos cambios; y el creciente protagonismo de los niveles de desempleo, una de sus manifestaciones más prominentes (2018). Finalmente, narrar cómo se fue difuminando la posibilidad de opciones laborales fuera de este mecanismo es parte de los objetivos de la apuesta de Dukes (2014; 2018).

El protagonismo de los niveles de empleo (y desempleo) en las representaciones institucionales y corporativas del mercado laboral en la Sabana, de espaldas a las limitantes que enfrentan las posibilidades laborales de las mujeres, son un ejemplo de lo que la sociología económica del derecho laboral de Ruth Dukes cuestiona. Es una manifestación de la forma en que se posiciona el aseguramiento de las condiciones económicas generales consideradas como propicias para el crecimiento del sector privado como mecanismo predilecto para la creación de empleo (Dukes, 2018). Las lecturas institucionales y de política pública de la alcaldía, el Concejo de Madrid y otras instituciones del orden nacional, en las que se tiene como principal objetivo de desarrollo el de hacer de Madrid "un territorio fuertemente atractivo para la inversión” (Acuerdo 024, 2000, art. 27), y el desempleo como una tasa a 
disminuir lo máximo posible sin ninguna consideración relacional y territorial, son plenos reflejos de una lectura reduccionista del trabajo en el marco del paradigma del desarrollo periurbano.

Ahora, si bien su propuesta se centra en el contrato de trabajo como objeto de análisis, en este artículo se extienden sus preguntas sobre el control democrático a dinámicas globales que han dado lugar a formas de trabajo y subsistencia que no entran, usualmente, en las aproximaciones tradicionales al derecho laboral. Además, el artículo se apoya en la crítica de Dukes a la centralidad que las condiciones económicas generales -consideradas como propicias para el crecimiento del sector privado- han tenido en el derecho laboral en un escenario donde el trabajo solidario se disputa el significado y destino de un territorio periurbano. El objeto es proponer lo territorial como aspecto relevante para una sociología económica del trabajo a partir de la incorporación de elementos de la ecología política feminista y la geografía jurídica crítica, que han confluido en las políticas de lugar desarrolladas por Asoquimad y la EMM para la defensa de su proyecto de vida.

Las delimitantes territoriales del trabajo propuesto más adelante, desde estos lentes, hacen visibles, de un lado, la presencia de presiones económicas locales y globales, y del otro, la ausencia de deliberación democrática en la organización territorial que demarca las opciones laborales y políticas de las mujeres. Esto implica aceptar que el derecho laboral se constituye como un campo en disputa en el que, cada vez más, se van reduciendo los espacios de deliberación y de control democrático, y superar el mito liberal del mercado laboral como espacio libre donde los agentes se encuentran para intercambiar la fuerza de trabajo por un precio. En pocas palabras, evita la descripción y normalización de lo laboral como apolítico (Dukes, 2014).

Este segundo componente metodológico es resultado de la articulación de una apuesta por la coproducción de conocimiento de los movimientos sociales, desde una perspectiva feminista, con la investigación sobre su vivencia y comprensión del territorio, en este caso Asoquimad. De hecho, una de nosotras participa activamente del colectivo por medio de la Asociación Herrera, con la que se ha construido el tránsito hacia formas de trabajo alternativas en torno a las huertas de cultivo orgánico. Esta apuesta, además, responde a varios años de trabajo conjunto que ha permitido comprender el lugar central de los significados y materialidad en disputa de la idea del territorio. Este último punto está enmarcado en recientes y álgidas discusiones nacionales y regionales (Sandoval; Robertsdotter; Paredes, 2017), pero también externas (Halvorsen, 2018), sobre el alcance y vivencia del territorio.

Bajo estas premisas, se sigue una aproximación cualitativa que combina el trabajo documental de revisión de literatura institucional, local y nacional, sobre los cambios en el uso del suelo, entrevistas semiestructuradas, historias de vida, 
reconstrucción de la historia jurídica de las discusiones sobre el terreno realizada por las mujeres de la Asociación Herrera, conversaciones informales, así como recopilación de información y percepciones sobre el territorio mediante grupos de discusión y mingas de trabajo comunitario realizadas en el terreno y en huertas caseras. Es a través de la articulación de esta aproximación cualitativa que se proponen y abordan las determinantes territoriales de las opciones laborales de estas mujeres en el escenario periurbano donde intentan hacer un tránsito del trabajo en la agroindustria de las flores a trabajadoras cooperativas. El objetivo es que esta aproximación, que continúa en curso, contribuya a hacer visibles las experiencias de las mujeres, y sus demandas laborales y territoriales, al tiempo que promueva un constate ejercicio reflexivo por parte de la academia con relación a la investigación con y sobre movimientos sociales.

\section{Mucho más que un terreno "prestado"}

Asoquimad es una organización conformada mayoritariamente por mujeres que trabajaron y sembraron hortalizas y verduras de manera orgánica en un predio de propiedad de la Alcaldía de Madrid, ubicado en la vereda Los Árboles, por cerca de siete años. Esta propuesta surgió, en un principio, dentro del marco de un programa municipal, con el apoyo de recursos de la ONG Visión Mundial, que tenía como propósito generar tres colectivos de mujeres, de los que no existe ninguno en la actualidad.

Un grupo de doce mujeres, la mayoría madres cabeza de familia y extrabajadoras de empresas de flores, decidió asociarse y trabajar en el terreno un proyecto de huerta orgánica y recuperación de la semilla de quinua como producto de gran valor nutricional y ancestral de la región. Al inicio, con el apoyo de la administración y Visión Mundial y, posteriormente, de manera más autónoma. El apoyo gubernamental consistía en transportar a las mujeres de la zona urbana a la rural y llevar agua con un carro tanque y combustible para una bomba que las mujeres gestionaron. Luego de varios meses de funcionamiento del proyecto, la administración cesó estos apoyos y varias mujeres se retiraron por los costos de los transportes. No obstante, otras mujeres decidieron continuar, al tiempo que-como se indicará más adelante-establecían relaciones con múltiples organizaciones con quienes gestionaron donaciones para agricultura y transformación de alimentos, talleres, asistencia técnica y redes para promover la soberanía alimentaria.

Este proceso ha sido entendido y desarrollado por las mujeres como un conjunto de acciones colectivas de lugar para la transformación de sus vidas, pero también "de la lógica de desarrollo que prevalece en el territorio" (Flórez; Lara; Veloza; Cardozo; 
Espejo, 2017: 40). Para entender las motivaciones y circunstancias que llevaron a la conformación de Asoquimad, así como la manera en la que el territorio y el trabajo están relacionados, resulta imprescindible un contexto de movilización social más amplia y, en particular, los proyectos y acciones llevados a cabo por la Asociación Herrera y la EMM.

La Asociación Herrera es una organización social que surge de la iniciativa de hijas e hijos de personas trabajadoras y extrabajadoras de empresas de flores que empezaron a realizar una lectura crítica del territorio a partir de sus problemáticas sociales y ambientales. En alianza con otras organizaciones, decidieron apostarle a permanecer y defender el territorio mediante la organización social con mujeres y jóvenes trabajadores y extrabajadores de empresas de flores de la Sabana Occidente, principalmente en Madrid. La EMM, por su parte, nació en 2011, como proyecto central de la Asociación Herrera, en educación popular en derechos humanos. Este es un espacio y proyecto pedagógico para el encuentro intergeneracional de mujeres populares, y para entender cómo la agroindustria y los proyectos de desarrollo en el territorio sabanero se han "instalado en la vida de las mujeres" (Flórez et al., 2017:36). Como colectivo, está conformada por mujeres y hombres trabajadores y extrabajadores de flores, sus hijas y algunos de sus hijos, amas de casa, empleadas domésticas, cuidadoras y otras mujeres que trabajan en la economía informal del municipio.

Las actividades y orientación de la asociación y la escuela articulan el territorio y el trabajo alrededor de dos ejes de acción interconectados: constituir un referente intergeneracional para potenciar el tránsito de las mujeres en la Sabana Occidente -principal, pero no solamente trabajadoras y extrabajadoras de la industria de flores- a trabajadoras cooperativas, y situar la corporalidad en el centro de la lectura del territorio y el entorno. En este marco, se desarrolló la apuesta de Asoquimad, la cual implicaba mucho más que cultivar un terreno cedido por la administración local. Era la concreción del tránsito a una economía solidaria de baja escala para la producción de alimentos que concretaba a su vez una apuesta por la defensa de la vocación campesina del suelo y una manera de resistir el tipo de desarrollo que subyace al rápido crecimiento periurbano de Madrid-de cuyo diseño no habían sido parte-. En este sentido, la siembra colectiva del terreno, el encuentro cotidiano en el mismo para cultivar, compartir experiencias y saberes, y su uso como laboratorio para la economía campesina de pequeña escala les permitía relacionarse de otra manera con un paisaje cambiante, así como ofrecer un lugar de encuentro a otras personas interesadas en promover la autonomía alimentaria en la Sabana.

En 2009, la alcaldía permitió el uso de cerca de $2000 \mathrm{~m}^{2}$ a las mujeres de Asoquimad, donde ellas trabajaron la tierra para formar una huerta de hortalizas y plantulero. Estas mujeres convirtieron el terreno en un espacio donde no solo cultivaban 
alimentos, sino que difundían conocimientos agroecológicos entre ellas con la práctica del cultivo cotidiano, intercambiaban experiencias con otros movimientos sociales y discutían las posibilidades de la autonomía alimentaria en la Sabana con otras organizaciones y habitantes, hasta que, en 2014, según lo narran las mujeres, la alcaldía decidió reducir el uso a $1000 \mathrm{~m}^{2}$. Con esta reducción, también llegaron decisiones de la administración para disciplinar el trabajo en la huerta. Para ello, una persona fue encargada de registrar los horarios de salida y entrada de las mujeres, así como las actividades realizadas. A partir de este momento, inició un largo intercambio entre Asoquimad y diferentes funcionarios con el fin de entender qué ocurriría con el terreno, por qué se redujo su extensión y, luego de ser expulsadas en noviembre de 2015, qué posibilidades tenían de retornar para mantener su apuesta cooperativa, política y agroecológica.

Las mujeres de Asoquimad y de la EMM sabían, desde el comienzo, que el proceso social que adelantaban probablemente daría lugar a tensiones y largos desacuerdos con autoridades locales y nacionales en el contexto de la disputa por el alcance y significado del desarrollo. Lo anterior debido, principalmente, a las implicaciones que megaproyectos como la zona franca de Mosquera, los monocultivos o la construcción del nuevo aeropuerto podían tener sobre los usos del suelo, el medio ambiente, la vida cotidiana y las posibilidades para la agricultura en la Sabana. Lo que no imaginaron fue lo intricado y agobiante que sería disputar el uso de $2000 \mathrm{~m}^{2}$ de un terreno de la alcaldía que ni siquiera era parte de estos proyectos. Tampoco pensaron que ese terreno o, mejor, su imaginaria destinación en documentos y alocuciones, siguiera determinando sus posibilidades para consolidar un proyecto de vida solidario y campesino, incluso mucho después de haberlo perdido.

Entre 2014 y 2018, las mujeres de Asoquimad, junto con la Asociación Herrera, solicitaron en múltiples ocasiones reuniones con funcionarios de la alcaldía con la finalidad de continuar haciendo uso del terreno, entender cuál había de ser la destinación del mismo e, incluso, solicitar el retorno de materiales y equipos que les pertenecían. Muchas de las solicitudes no recibieron respuesta, otras fueron tratadas de forma ambigua, y otras se ahogaron en procesos interminables para el cumplimiento de formalidades y requisitos insólitos (como puede evidenciarse en los documentos que reposan en el archivo de la asociación). A continuación, se hará referencia, específicamente, a las discusiones sobre la destinación y denominación del terreno.

Durante varias reuniones, en 2016, diferentes funcionarios de la alcaldía hicieron constantes referencias a la meta 189 del Plan de Desarrollo para justificar por qué tuvieron que "sacarlas del terreno". La meta, incluida en el eje "Madrid ciudad emergente, ciudad agropecuaria" -título que no deja de ser paradójico-, señala: 
"MANTENER en funcionamiento de la Granja demostrativa"6. Aparte de esto, el plan no indica nada más, por lo cual no permite saber a qué predio se refiere $y$, de ser el terreno en cuestión, por qué ha sido denominado así. En las reuniones, no obstante, los funcionarios insistieron en que Asoquimad no podía continuar con su proyecto, pues no era una "granja demostrativa". Pero antes de la expedición del plan, donde dicho término aparece por primera vez formalmente, el proyecto de la organización en el terreno fue reconocido por la institucionalidad, como consta en la certificación del 28 de junio de 2013, firmada por el entonces secretario de Desarrollo Económico y Social del municipio, como un proyecto productivo "con énfasis en Agricultura Orgánica".

Las mujeres, confundidas, insistían en que se les explicara qué era una granja demostrativa y por qué no podían encargarse de la misma mientras mantenían su proyecto. Ante la confusión, el 7 de octubre de 2016, Asoquimad interpuso un derecho de petición en el que solicitaron, al alcalde de Madrid y al director de Fomento Agropecuario, información sobre la destinación que se dio al "terreno, granja demostrativa vereda Los Árboles”, ubicado en "el camellón Fresas San Ramón, al lado del polideportivo", así como una reunión con ambos funcionarios. Específicamente, preguntaron si la meta 189 del Plan de Desarrollo "quería decir que le darán continuidad a los proyectos que venían funcionando allí”, quiénes lo trabajarían, qué asociaciones participarían y, en tal caso, qué procedimiento se emplearía para elegir la propuesta.

En su respuesta, la alcaldía explicó el objetivo de la meta 189 y definió lo que entendía por granja demostrativa. El objetivo era "brindar un espacio a pequeños y medianos productores, instituciones educativas y personas interesadas en el sector agropecuario para impulsar y difundir la importancia de la Granja Integral como una alternativa de producción agroecológica". Y definía las granjas integrales como "sistemas de producción de alimentos para el autoconsumo que contribuyen a mejorar la seguridad alimentaria y la economía de los agricultores que son capacitados en ellas. Además de reparar, adecuar e implementar sistemas para el correcto funcionamiento de la misma y poder realizar charlas fomentando la integración social por medio de talleres participativos y de visitas guiadas en los módulos demostrativos". La respuesta no aclaró por qué Asoquimad no podía realizar los objetivos allí descritos. Y, en todo caso, dicha granja no ha entrado en funcionamiento.

Aunque el terreno no ha sido usado, sí ha sido ocupado discursivamente. Tanto en la respuesta al derecho de petición como en diferentes actuaciones, la administración se ha referido a la granja como si estuviera en funcionamiento, como si existiera. El 
22 de agosto de 2016, la Secretaría de Desarrollo Económico del municipio (2016) presentó un estudio técnico - del que no informó a Asoquimad-para la celebración de un contrato relacionado con la denominada granja. En la justificación de dicho contrato, la alcaldía señalaba que su finalidad era dar cumplimiento al Plan de Desarrollo, específicamente al objetivo de "mantener en funcionamiento la Granja demostrativa". No obstante, al momento de referirse al objeto del contrato que se celebraría, el estudio técnico hablaba del "servicio de mantenimiento del invernadero y un reservorio de la granja integral demostrativa", es decir, no se trataba de un servicio para operar la granja.

En la respuesta al derecho de petición, la alcaldía informó a Asoquimad que los operadores de la granja serían contratados por el municipio, lo cual daba la impresión de que la contratación tendría por objeto el funcionamiento de la aquella. Sin embargo, tanto el estudio técnico como el contrato establecieron que su objeto era realizar un mantenimiento, por quince días hábiles, del "invernadero y un reservorio de la granja integral demostrativa", granja que, a menos que se entendiera que lo que había hecho Asoquimad era una granja demostrativa, no existía. El contrato fue celebrado el 31 de octubre de 2017 por una suma de \$20.500.00o, inició el 2 de noviembre siguiente y terminó el 21 del mismo mes. Entre el estudio y el contrato, el terreno permaneció sin uso, así como después del 21 de noviembre. Entonces, la alcaldía no pudo mantener en funcionamiento una granja, primero, porque nunca inició operaciones, y segundo, porque celebró un contrato por solo quince días para el mantenimiento del terreno, mas no para el funcionamiento de granja alguna.

Es difícil saber si la reticencia del gobierno local a permitir la continuidad del proyecto de Asoquimad se debe realmente a un compromiso con una idea de desarrollo que no pudo cumplir -la conformación de una granja emblemática de una ciudad emergente, pero también agropecuaria-, a la ineficiencia de la administración, a una oposición al proyecto de autonomía alimentaria y organización solidaria de las mujeres por contradecir la visión de desarrollo del municipio o, simplemente, a un cinismo institucional. En el derecho de petición antes mencionado, la asociación preguntó a la alcaldía si conocía o tenía información sobre los procesos de soberanía alimentaria que se adelantaban en el municipio y de los que hacía parte. El silencio del gobierno local frente a esta pregunta se acerca a las dos últimas opciones.

La pregunta era, quizás, retórica, en la medida en que las iniciativas por la autonomía alimentaria, incluyendo la de Asoquimad, eran y son un proyecto reconocido en la Sabana Occidente. Pero el silencio al respecto y las confusas invocaciones a una granja inexistente sin duda contribuyeron a invisibilizar la apuesta laboral y territorial de la asociación. Además, las respuestas elusivas y vagas de la administración, hasta hoy, pasaron a constituir una parte significativa de la cotidianeidad de las mujeres. 
Actualmente, ellas se debaten entre peticiones para obtener la entrega de equipos e instrumentos de cultivo que les fueron donados tiempo atrás -que permanecen en la alcaldía- y promesas disciplinantes que condicionan la posibilidad de volver al terreno a su "buen comportamiento", el cual consiste en asegurar el éxito de diferentes iniciativas de la alcaldía (mujer miembro de Asoquimad, comunicación personal, 10.2018).

Así, la pérdida del terreno representa una ruptura de muchos más procesos que el solo cultivo del mismo. Lo que comenzó como un intento de disciplinar los cuerpos y tiempos de las mujeres, a través de su administración, terminó en la historia de un terreno hiperadministrado, pero físicamente abandonado. Esta disputa por un pequeño terreno que, no obstante, era la posibilidad material de concretar una opción laboral, política y territorial, no puede desvincularse de las transformaciones del entorno en el que ocurre. A continuación, se explicará el carácter periurbano de la Sabana de Bogotá para, en ese escenario, leer la apuesta de Asoquimad y de las dificultades que enfrentan hasta hoy, a través de un conjunto de dimensiones territoriales de su trabajo en un territorio de ese carácter.

\section{La ubicación periurbana}

La ubicación de Madrid, en las afueras de Bogotá, ha jugado un papel definitivo en las transformaciones del territorio y el paisaje, así como en las opciones y horizontes laborales de sus habitantes. En este artículo, se sugiere que esta especial ubicación, junto con los rápidos cambios asociados a ella, corresponden a lo que se ha denominado como periurbanización. En términos generales, esta expresión hace referencia a "los procesos transformadores que ocurren en las áreas que rodean a grandes ciudades" (Friedmann, 2016:163)7. Regularmente, por áreas periurbanas y periurbanización se hace alusión a procesos de cambio en sitios que no pueden ser completamente comprendidos ni como urbanos ni rurales; estos procesos pueden incluir la emergencia de construcciones urbanas en áreas rurales semiperiféricas como, por ejemplo, distritos industriales. Asimismo, las áreas periurbanas son denominadas, por ejemplo, como suburbios en espera bajo la suposición de que terminarán por ser urbanizadas; como "terceros espacios" entre lo urbano y lo rural con funciones y características particulares; o, en general, como "espacios en mutación" (Ruoso; Plant, 2018).

7. Traducción propia. 
La abundante y compleja literatura sobre estas áreas refleja "las dificultades de capturar estos procesos de movimiento rápido, y la variada diversidad de experiencias en diferentes escenarios" (Sorensen, 2016: 134). No obstante, es posible identificar ciertas características en común. Primero, son áreas comúnmente ubicadas en las periferias de las ciudades que atraviesan "cambios en el uso del suelo asociados a la proximidad de la ciudad, y primordialmente cambios de suelo rural a usos urbanos" (Sorensen, 2016: 134; veáse también Chadha; Sen; Sharma, 2004). Estos usualmente son cambios del suelo para la agricultura a usos residenciales e industriales, pero también de las formas e intensidad en que se han de realizar las actividades de agricultura (Ruoso; Plant, 2018) ${ }^{8}$. Además, en estas zonas tienen lugar conflictos sobre qué constituye "lo rural" (Ruoso; Plant, 2018), el desarrollo, las disputas por recursos entre la ciudad y estas áreas (Díaz-Caravantes, 2012), y sobre quién define y cómo los cambios del paisaje o qué prácticas son consideradas más legítimas (Ruoso; Plant, 2018).

La conexión entre las áreas periurbanas y el trabajo, por su parte, ha sido abordada desde diferentes y contrastantes perspectivas. El Banco Mundial (2009), por ejemplo, ha indicado que, si bien los rápidos cambios en las áreas periurbanas vienen acompañados de unas desigualdades regionales iniciales, con el tiempo la oferta de empleo termina por estar disponible para los habitantes. Así, se espera que se generen nuevas y diversificadas oportunidades de trabajo, como parte de lo que denomina "desarrollo inclusivo" (Banco Mundial, 2009). En contraste, otras aproximaciones más críticas han visibilizado cómo estas áreas están expuestas a múltiples y diversas vulnerabilidades, a la exclusión de las poblaciones de las oportunidades asociadas con el fenómeno y a las cargas que la expectativa de rápido tránsito hacia modos de vida no agrarios impone a las poblaciones rurales (Sen, 2016).

Específicamente, la situación de las mujeres en estas áreas ha sido también objeto de esta perspectiva crítica. Sucharita Sen (2016), por ejemplo, ha abordado las diferencias de género en la participación laboral de las mujeres en áreas periurbanas de India y, en particular, el tipo de barreras, las diferencias salariales y la clase de trabajos a los que acceden. Silvia Chant y Kerrwin Datu (2015) han señalado que cuando la prosperidad es considerada en conjunto con la pobreza, las inequidades de género se hacen más evidentes en dinámicas de urbanización. Así, enfatizan la importancia de problematizar la manera en que la prosperidad es abordada analíticamente, en particular, de qué manera la riqueza es acumulada en las ciudades,

8. El cambio en los usos del suelo respecto a la agricultura puede enmarcarse en dos grandes y contrastantes caminos: de un lado, "las actividades de agricultura se tornan crecientemente industrializadas e intensivas"; del otro, las transformaciones consisten en "un giro hacia un paisaje post-productivista y multi-funcional" (Ruoso; Plant, 2018: 58). 
cómo son usados los datos macro estadísticos, y qué ocurre cuando observamos estos factores a través de "los lentes de género" (Chant; Datu, 2015).

La producción de alimentos, asimismo, ha sido un referente importante entre las aproximaciones a la relación de áreas periurbanas y trabajo. Con frecuencia, estas hacen alusión al término "sombrilla de Agricultura Urbana (AU)". Entre las más difundidas, se encuentran las políticas para el desarrollo y la planeación de ciudades sostenibles (Smit; Ratta; Nasr, 2001); las que enfatizan las tensiones entre la producción periurbana de alimentos en pequeña escala y la resistencia de los gobiernos locales que consideran esta posibilidad como perjudicial para su desarrollo e imagen o simplemente no apoyan esta alternativa (Tomaghi, 2014); aquellas que enfatizan las dimensiones políticas de las huertas urbanas en estas áreas como forma de resistencia a la producción industrial de alimentos (Wekerle; Classens, 2018); y otras perspectivas que identifican los casos de inclusión de las huertas urbanas en programas públicos como parte de la provisión de alimentos y de contribución a la economía de las ciudades adyacentes.

Ahora, en relación con Madrid, en particular, el establecimiento de una zona franca $^{9}$ en Mosquera -municipio vecino- y el actual proyecto de construcción de un segundo aeropuerto para Bogotá constituyen dos de las grandes causas de las transformaciones más recientes del uso del suelo. Ambas son resultado de políticas y proyectos de desarrollo diseñados para esta área reflejando el rol central de la capital, el nivel nacional en su diseño y propósito, y, en últimas, el carácter periurbano de la región. Estas iniciativas permiten, además, comprender la intensificación de la disminución de la tierra cultivable en la Sabana y en Madrid en particular, al tiempo que evidencian el carácter debatido del tipo de desarrollo que impulsan.

La zona franca de Mosquera fue aprobada en diciembre de 2008, mediante una resolución que declaró 32 hectáreas como zona franca permanente. Está estratégicamente ubicada en el municipio de Mosquera, a $12 \mathrm{~km}$ de Bogotá y $20 \mathrm{~km}$ del Aeropuerto Internacional El Dorado. En efecto, se encuentra muy cerca de infraestructura en servicios y vías que conectan con puertos, aeropuertos y zonas fronterizas, lo cual facilita su articulación con los circuitos comerciales globales. Está además conectada con el centro del país al encontrarse situada frente a la carretera Troncal Nacional de Occidente, variante Madrid, vía que conecta desde el

9. Las zonas francas son áreas administrativas especiales donde las empresas gozan de un tratamiento tributario y aduanero especial. La Ley 1004 de 2005, "Nuevo Régimen de Zonas Francas", en su Artículo 1. señala que son un área geográfica "en donde se desarrollan actividades industriales de bienes y servicios, o actividades comerciales, bajo una normatividad especial en materia tributaria, aduanera y de comercio exterior. Las mercancías ingresadas en estas zonas se consideran fuera del territorio aduanero nacional para efectos de los impuestos a las importaciones y a las exportaciones". El proceso de la zona franca de Mosquera fue objeto de múltiples cuestionamientos cuyo análisis excede el objeto de este artículo. 
municipio de Honda con los puertos de Buenaventura, sobre el Océano Pacifico, y Turbo, Cartagena, Barranquilla y Riohacha, sobre el Atlántico.

Más allá de la proyección de empleos en la zona franca ${ }^{10}$, no contamos con estudios que aborden la manera en que esta ha impactado el trabajo en la Sabana Occidente en particular, las opciones laborales y la relación con el territorio. Para las mujeres de Asoquimad y de la EMM, este puerto seco no solo hace más difícil la agricultura en la región sino que no se ha traducido en mejores condiciones laborales para las mujeres en general de Sabana Occidente. Sobre este punto, vale la pena resaltar que aunque son promocionadas como generadoras de empleo o instrumentos para su creación, diversos sectores han expresado dudas acerca de la real incidencia de las zonas francas en este sentido. Por su parte, la nueva etapa del Aeropuerto El Dorado II estaría ubicada entre Facatativá y Madrid (Unidad Administrativa Especial Aeronáutica Civil, 2013). Actualmente, está en etapa de estructuración y la proyección de la nueva etapa atravesaría al humedal Moyano ${ }^{11} \mathrm{y}$ varios cuerpos de agua y zonas de cultivo. Para Asoquimad y las organizaciones sociales de la Sabana en general, el nuevo aeropuerto reduciría aún más el suelo disponible para la agricultura además de afectar el acceso al agua y al entorno.

El carácter periurbano de Madrid es innegable. Sin embargo, la iniciativa de Asoquimad no puede enmarcarse como parte de un proyecto de desarrollo periurbano para la producción de alimentos ni tampoco puede leerse en las coordenadas de huertas urbanas en los términos de la literatura más difundida. Si bien la apuesta de las mujeres de la asociación por volver a la tierra, es decir, por volver a la siembra colectiva en defensa de la vocación campesina del suelo y en resistencia al modelo de desarrollo que subyace a la periurbanidad, responde a una preocupación por la producción de alimentos, la cual constituye un eje central de la literatura sobre $\mathrm{AU}$ en áreas periurbanas, esta es inseparable de la historia laboral y corporal de las mujeres, de la transformación del territorio y de las condiciones materiales que enfrentan para iniciar y mantener este tipo de iniciativas. Estas características exigen un análisis diferente al de la literatura más reciente sobre la relación entre territorio $\mathrm{y}$ trabajo. A continuación, proponemos una serie de determinantes territoriales del trabajo de las mujeres en Madrid.

10. Las zonas francas permanentes son actualmente 38; y a junio de 2016 generaban 50.247 empleos directos y 81.232 indirectos en el país (Asociación de Zonas Francas de las Américas, 2017). No se cuenta con información desagregada acerca del número de empleos generados por la zona franca de Mosquera, en particular.

11. Para una descripción de la discusión sobre el humedal Moyano, ver Asociación Ambiente y Sociedad (2017). 


\section{Dimensiones territoriales del trabajo en zonas periurbanas}

En este apartado sugerimos una serie de dimensiones territoriales del trabajo a partir del caso de Asoquimad. El carácter periurbano de la Sabana Occidente y de Madrid, en particular, ha jugado un papel determinante tanto en la configuración de las opciones laborales como en la relación entre las transformaciones del territorio y la manera de abordar la idea misma de trabajo en la Sabana Occidente. En este marco, presentamos una serie de determinantes territoriales del trabajo, que incluye tres articulaciones específicas entre trabajo y territorio, identificadas por las mujeres de esta organización, y dos de los procesos de transformación del territorio y de los usos del suelo que más incidencia tuvieron en la apuesta cooperativa de la asociación. Si bien todos los determinantes identificados están interrelacionados, cada uno dice cosas específicas sobre la relación trabajo-territorio.

\section{La centralidad de las trayectorias y horizontes laborales de las mujeres}

Las trayectorias laborales de las mujeres que hacen parte de la EMM y de Asoquimad jugaron un papel decisivo para su conformación. Como muchas de las habitantes de la Sabana de Bogotá, en algún momento de su vida trabajaron en el cultivo agroindustrial de flores o tuvieron familiares que lo hicieron. La decisión de organizarse en el proyecto de cultivo colectivo y autónomo para la producción local de alimentos respondió en gran medida a la historia de trabajadoras que "luego de salir como 'desechadas' de la industria de las flores" (mujer miembro de Asoquimad, comunicación personal, 05.2015) o tras abandonar su trabajo allí decidieron enfrentar un mercado laboral con pocas opciones. Sin embargo, es importante resaltar que la relación con la floricultura no es simple. Si bien las mujeres de Asoquimad y de la EMM reconocen y cuestionan las marcas que este trabajo dejó en sus cuerpos y territorio, también afirman que fue su fuente principal -o única- de ingresos:

La Sabana cambió su modo de vida campesino. La floricultura rompió las formas campesinas de relacionamiento con el territorio, la tierra y el entorno en desconocimiento de otras formas de producción que permitieran mantener esas formas de relación. Además, la tierra y las mujeres trabajadoras entraron de una manera subordinada a la industria floricultura. Sobre esto último es importante resaltar, y es un tema difícil, que el hecho de que las mujeres entraran al mundo laboral no implicó una emancipación. Si bien muchas mujeres empezaron a obtener un ingreso económico, ello vino acompañado de una precarización expansiva, contemporáneamente traducida en medidas de flexibilización laboral. Es muy difícil hablar de esto, pero insistimos que podría y tendría que ser de otra manera (mujer miembro de Asoquimad, comunicación personal, 12.2014). 
Las posibilidades laborales también modelaron la apuesta de Asoquimad. En el cambio generacional las personas tienen tres opciones laborales delimitadas por el tipo de desarrollo y productividad prevalente en la Sabana: continuar trabajando en la flora, que para ellas representa un "panorama de desolación"; migrar a Bogotá, donde hay mayor competencia laboral pero mejores posibilidades de educación; o "crear nuevas opciones de vida y laborales basadas en su territorio" (Flórez et al., 2017: 26; mujer miembro de Asoquimad, comunicación personal, 10.2014; 14.12.2014). Las dos primeras impiden o al menos dificultan en gran medida la posibilidad de optar por la tercera.

\section{El continuum cuerpo-territorio}

Un eje central del trabajo de la Escuela de Mujeres y de Asoquimad es el papel del cuerpo para comprender la relación entre el trabajo y el territorio como un lugar en disputa. Para estas mujeres, "[1] a transformación de los cuerpos de las trabajadoras no puede separarse de la transformación del territorio" (Flórez et al., 2017:34). Las transformaciones espaciales de la Sabana Occidente por causa de la agroindustria de flores y, más recientemente, la declaración de una zona franca en el vecino municipio de Mosquera, la construcción de la variante de Madrid y la planeación de un nuevo aeropuerto para Bogotá, han trasformado las interpelaciones entre el cuerpo de las mujeres y su entorno territorial.

En particular, las rutinas de la agroindustria de las flores, como consecuencia de realizar durante años ejercicios mecánicos, han modelado sus cuerpos, hábitos e incluso sus movimientos. El cuerpo de las trabajadoras habla y dice cosas sobre el territorio, pero también sobre la propia trayectoria laboral. Por ejemplo, ellas resaltan cómo sus cuerpos están impregnados de un fuerte olor a caucho y azufre que hace que adviertan su presencia donde quiera que estén (Veloza; Lara, 2014; mujer miembro de Asoquimad, comunicación personal, 12.2014); también evidencian con frecuencia marcas indelebles en sus uñas y piel como consecuencia de años de interacción con pesticidas y químicos mientras trabajaban bajo el sol (Flórez et al., 2017). Estas son historias del territorio que están presentes en sus cuerpos, incluso luego de que las empresas modificasen el tipo de pesticidas y varias de las prácticas empleadas. Adicionalmente, las rutinas de la agroindustria también han transformado su relación cotidiana con el entorno. Por ejemplo, el tránsito constante de los camiones de transporte de flores en las carreteras locales ha llevado a una lucha diaria de los hogares con el polvo que invade su privacidad, cuerpos y huertas caseras.

Así, las trayectorias laborales de participación en la cadena productiva de la industria floricultura marcaron de forma abrupta el cuerpo de las mujeres que parti- 
ciparon tanto en los cultivos como en la poscosecha, donde el horario laboral era de entre ocho y once o catorce horas, respectivamente, y podía alcanzar las dieciocho horas (Lara; Veloza; Flórez, 2015). Debido a los movimientos físicos constantes y repetitivos que las mujeres realizaban como parte ordinaria de su trabajo con las flores, se configuró en ellas una corporalidad alienada que es en sí misma expresión de la enajenación territorial que implicó el trabajo asalariado (Lara et al., 2015). La transformación del cuerpo no es ajena a la del territorio; en el cuerpo de las mujeres de Asoquimad se imprimió la supeditación del trabajo al capital, y una corporalidad del trabajo anclada al dolor silencioso. Aún después de haber dejado sus trabajos anteriores sus cuerpos recuerdan las vivencias de esa condición pasada.

Por su parte, la EMM ha articulado la relación entre el cuerpo y el territorio con la educación por el trabajo digno a través de una propuesta pedagógica (Flórez et al., 2017). Este proyecto educativo popular, que subyace también a la apuesta de Asoquimad, está basado en el reconocimiento de "la conexión entre educación, trabajo y economía" y en la búsqueda de alternativas para el continuum cuerpo-territorio más allá de las opciones ofrecidas actualmente en la Sabana Occidente. Una política del lugar inspirada en la contribución central del feminismo que emerge de la confluencia de los estudios críticos del desarrollo y la geografía está en el eje de la propuesta (Flórez et al., 2017). Esta hace referencia al conocimiento de las mujeres respecto al lugar que comparten, a la creación de espacios fuera de la proyección desarrollista que ven manifestada en los monocultivos y la zona franca y, específicamente, en "lugares de trabajo alternativos a los ofrecidos por la floricultura" (Flórez et al., 2017:42).

La conexión entre el cuerpo y el territorio para las mujeres de Asoquimad está mediada por la forma de trabajo cooperativo que subyace a su apuesta por la reapropiación del cuerpo, el trabajo y el territorio. A través de su labor en la huerta orgánica se abrió paso una experiencia corporal más conectada con el territorio, que es consciente de que en el trabajo su cuerpo no debe estar anclado al dolor y al sacrificio (Lara et al., 2015). Así, es posible reconfigurar el territorio con cultivos de autoaprovisionamiento, técnicas de cultivo orgánico y alternativas al trabajo en bodegas o flores, a medida que las mujeres se reapropian y concientizan de las vivencias de sus cuerpos. En efecto, "aunque la productividad sigue estando en el centro de los cultivos, las mujeres dejan atrás el estrés de producir según metas establecidas por otros, discuten el trabajo por realizar, negocian metas y ritmos laborales según sus capacidades, dolencias, enfermedades y necesidades familiares" (Flórez et al., 2015: 208). 


\section{La disputa por "lo rural"}

En medio de las rápidas transformaciones de la Sabana Occidente, el proyecto de las mujeres de Asoquimad se disputa también la representación, materialidad y significado de lo rural y lo campesino en un escenario periurbano ${ }^{12}$. El cultivo orgánico en el terreno, las actividades de transmisión del conocimiento, y la recuperación de las flores nativas eran acciones de la asociación que cuestionaban, de un lado, la idea de una ruralidad lejana, y del otro, un tipo de desarrollo que hasta hoy continúa decidiendo la transformación del territorio y limitando las posibilidades de una economía solidaria alrededor de la pequeña agricultura.

Con relación a la ruralidad lejana, en el terreno disputado las mujeres buscaban modificar una relación con lo rural mediada por lo que algunas han denominado "lejanías":

Su relación con el entorno está delimitada por las "lejanías". Todo, excepto el pueblo y la ruta del bus que lleva las trabajadoras a la empresa, es una lejanía construida por sus horarios laborales y el cansancio que las domina en épocas de gran producción -navidad y San Valentín-. La mayoría de las trabajadoras vive en la parte urbana del municipio y su relación con el entorno rural esta mediada por el bus de la empresa que las transporta. Salen muy temprano y vuelven muy tarde. "La flora", nombre con el que se designa generalmente a las empresas de flores, domina su cotidianidad, pero no es concebida como parte del campo. Muchos no conocen el campo de Madrid. En nuestro trabajo con trabajadores y extrabajadores de la flora, advertimos cómo en el ordenamiento del espacio rural en el imaginario social solo existen el pueblo, Bogotá y la flora (mujer miembro de Asoquimad, comunicación personal, 12.2014).

12. Son múltiples las discusiones contemporáneas sobre lo rural y la ruralidad. Así, por ejemplo, algunas aproximaciones han abordado la problemática de la transformación de los usos del suelo desde la noción de la gentrificación de lo rural (Phillips, 2004); otras han cuestionado las implicaciones de la denominada nueva ruralidad (Pérez, 2001; Echeverri; Ribero, 2002); otros trabajos han cuestionado las dinámicas de urbanización sin ciudad o la diferencia entre lo rural y lo campesino, entre otros aspectos. En Colombia los debates además incorporan problemáticas relacionadas con la implementación del Acuerdo de Paz de La Habana y el alcance que tendrá lo rural en materia de crédito o las transformaciones que su significado tendrá en virtud de las construcciones jurídicas de fertilidad alrededor de las zonas de interés de desarrollo rural y económico (Corte Constitucional, C-077, 2017). Un análisis de estos debates excedería el alcance de este artículo. Aquí nos referiremos a la disputa por lo rural en términos de la lectura que el movimiento hace de la posibilidad de realizar un modo de vida campesino, entendido como la autonomía para cultivar y sobrevivir de su cultivo independientemente de las configuraciones institucionales y corporativas sobre nociones como desarrollo, productividad, competitividad o seguridad alimentaria. 
De otro lado, las discusiones sobre el desarrollo y la planeación de la Sabana Occidente, con ocasión de las transformaciones del uso del suelo, orientan las acciones de las mujeres para reconfigurar el terreno pero también entornos como las huertas caseras, que consideran lugares campesinos. En este sentido, atraviesan y delimitan lo que las mujeres de la EMM y de Asoquimad entienden por trabajo y opciones laborales. Lo que han denominado "urbanización continúa" motivó su decisión de identificar y examinar cómo ocurre el desarrollo en la región, qué tipo de desarrollo prevalece y a quién beneficia ${ }^{13}$. Por último, la lucha por lo rural también se ha manifestado en el intercambio translocal con otros movimientos, iniciativas y organizaciones para la defensa de la producción campesina de alimentos, el agua y el territorio en el Ariari (Meta) y Viotá (Cundinamarca).

\section{La planeación de lo local desde el centro y lo global}

La ordenación del territorio en Madrid, así como su planeación, están sujetas a decisiones nacionales y provenientes de Bogotá. Esta manifestación de lo periurbano en el ordenamiento del territorio y la planeación del desarrollo constituye otro de los delimitantes del trabajo de las mujeres en Madrid. De un lado, la modelación del territorio desde Bogotá a través de su Plan de Ordenamiento Territorial ha generado cambios importantes en la demanda de bodegas, sobre todo porque la eliminación de la zona industrial que existía dentro de la ciudad, junto con la escasez de terrenos para la construcción y, en especial, para la implementación de usos industriales, produjeron una baja oferta de zonas de almacenamiento de mercancías para el comercio. La zona franca de Mosquera responde a tal escasez. Por su parte, los cambios en el uso del suelo en Sabana Occidente son muestra de la significativa modelación del territorio desde el nivel nacional. Ejemplo de esto son los cambios en los usos del suelo en los municipios aledaños a la capital. En Mosquera, municipio vecino de Madrid, los cambios en el uso del suelo de rural a industrial han tenido un papel fundamental en la conformación de la zona franca. Esto evidencia una de las características de las zonas periurbanas, es decir, la particular configuración de

13. Este trabajo de identificación colectiva fue realizado durante el Encuentro Social y Popular de la Sabana donde confluyeron, entre otros, la Red Ecológica, Raíces de la Sabana y la Red Popular de Mujeres de la Sabana, quienes han articulado las organizaciones de la Sabana de Bogotá. Algunas de las participantes que trabajan tanto en la academia como en la movilización social escribieron los resultados de esa identificación en Flórez et al. (2017). 
un entramado de normas locales y nacionales necesarias para cambiar el uso rural del suelo ${ }^{14}$.

De otro lado, los planes de ordenamiento y desarrollo de Madrid desarticulan las variables ambientales y de planeación de las valoraciones laborales. En efecto, no tienen en cuenta o se separan del detrimento del suelo y el agotamiento de los recursos hídricos. Primero, el Plan de Desarrollo actual (2016-2020) proyecta a Madrid como una "Ciudad Emergente, ciudad agropecuaria" con la finalidad de anticipar "el crecimiento poblacional y urbanístico para prepararse para un desarrollo equilibrado, resiliente y sostenible del territorio" (Acuerdo o05, 2016, art. 9). Los objetivos de esta proyección evidencian que la idea de ciudad emergente está íntimamente asociada a "la movilidad multimodal", que incluye el aeropuerto alterno, el corredor vial y férreo, y la consolidación de polígonos estratégicos para el crecimiento económico.

Además, el empleo está pensado a partir del fomento de "habilidades, competencias técnicas y profesionales para el ingreso al mercado laboral" (Acuerdo oo5, 2016, art. 22). Siguiendo esa línea, las propuestas programáticas de la política pública de Madrid se localizan a sí mismas desde la gestión de crecimiento económico y productivo en la capacitación de mano de obra, habilidades técnicas y condiciones de productividad. Tal enfoque encaja con la lógica de desarrollo de las zonas periurbanas. Es muy diciente que la meta de resultado del plan en materia de empleo sea la de "disminuir en 2 puntos porcentuales (9) la tasa de desempleo del Municipio" (Acuerdo 005, 2016, art. 22). En concordancia, las acciones de la institucionalidad están avocadas a la capacitación para el empleo y el desarrollo humano, así como a la competitividad de pequeños negocios en el marco de la atracción a la inversión y el crecimiento de la industrialización.

Estas características del actual Plan de Desarrollo de Madrid evidencian la visión de que el camino correcto es aquel que se dirige hacia el desarrollo mediante la constitución e integración a un gran puerto seco, mientras que a su población

14. Solo para nombrar una parte del entramado de producción normativa para cambiar el uso del suelo a nivel local el Acuerdo 017 de 2006 revisó y ajustó el Plan Básico de Ordenamiento Territorial y aplicó el concepto de suelo suburbano al de suelo rural. Esto indica las problemáticas que subyacen a las disputas por lo rural ya que supone que el suelo rural no es urbanizable. El Decreto 053 de 2007 definió Siete Trojes como zona de expansión urbana y aumentó el índice máximo de ocupación del 50 al 75\%, para construcciones en predios de la futura zona franca. Luego, el Decreto 116 de 2007 adoptó el Plan Parcial Siete Trojes y otorgó uso industrial al suelo de expansión. Esto es problemático porque el suelo de expansión sin urbanizar no podía tener uso industrial. A nivel nacional, el Decreto 1069 de 2009 modificó los decretos 097 de 2006, 3600 de 2007 y 4066 de 2008, y respaldó el aumento del índice máximo de ocupación que el municipio había realizado del 50 al 75\%, a pesar de que el suelo era rural y el índice de ocupación debía ser restringido. 
se le atribuye una vocación naturalizada de mano de obra para el abastecimiento, directo e indirecto, de la demanda de actividades comerciales e industriales de inversión que responden a factores exógenos. Incluso, el Plan Local de Empleo del municipio excluye la posibilidad del retorno a la tierra; los incentivos y apoyo para el trabajo de la tierra son casi inexistentes, y la centralidad del aprovechamiento del clima de inversión preponderante se posiciona en detrimento de la importancia de la economía agrícola y autónoma (Ministerio del Trabajo, 2012).

Así, resultan claros dos aspectos respecto a la planeación municipal. Primero, la desarticulación entre los cambios territoriales y las demandas sociales, por la autonomía alimentaria. En este sentido, a pesar de la movilización de la Asociación Herrera, así como de otros actores, por una nutrición orgánica y local, el plan actual no hace mención a programas de producción de alimentos ni al retorno a la tierra ni se vincula la educación con programas de educación popular agroecológica. $\mathrm{Y}$, segundo, la desarticulación entre el acceso al mercado para la población, la forma en que se piensa lo laboral a nivel local y el trabajo campesino de la tierra.

Igualmente, el Plan de Desarrollo construye la idea de progreso sobre lo que implicará tanto la futura implementación del aeropuerto como la integración regional de la gran Sabana Occidente. De hecho, de acuerdo con el plan, mantener alianzas estratégicas alrededor de la implementación del aeropuerto es fundamental para la promoción del desarrollo (Acuerdo 005, 2016). Con ello, la visión institucional y programática es indiferente a las movilizaciones sociales en contra de este tipo de enfoque económico y social.

Adicionalmente, el ordenamiento territorial de Madrid ${ }^{15}$ también está proyectado a partir del Plan Maestro del Aeropuerto El Dorado II, lo que desarticula las opciones laborales de iniciativas como la de Asoquimad sobre la planeación del territorio (Acuerdo 024, 2000). A pesar de que las mujeres han puesto de presente la relación cuerpo-territorio como un eje central para la planeación territorial en el Consejo Territorial de Planeación, las reformas al ordenamiento no han respondido a este llamado. Esta problemática se une a la ausencia de participación comunitaria como principio rector del proceso de ordenamiento territorial, y a la ausencia de caracterización de los conflictos de uso, y sus dimensiones sociales. En este sentido, la planeación territorial no solo es pasiva frente al pasado laboral de las mujeres, sino que desarticula la planeación de la vivencia del territorio, así como de los mecanismos de supervivencia y permanencia de sus habitantes.

15. En el municipio de Madrid, Cundinamarca, se aprobó el Plan Básico de Ordenamiento Territorial en el año 2000 y ha sido objeto de modificaciones posteriores; la última de ellas en 2012. 


\section{El papel del gobierno local}

Las limitaciones institucionales para optar por el trabajo de cultivo colectivo y solidario -tal como argumentamos en la primera parte de este artículo- constituyen la última dimensión territorial del trabajo aquí propuesto porque jugaron un papel definitivo al cerrar la posibilidad de uso del terreno en disputa. En conjunto, las dimensiones mencionadas dan un punto de entrada para conectar las transformaciones del territorio en la Sabana de Bogotá con las opciones laborales de las mujeres más allá de una lectura plana desde las cifras de creación de empleo.

La relación entre trabajo y territorio manifestada en la apuesta de Asoquimad y de la EMM exige pensar situadamente el trabajo a partir de una historia de las transformaciones del territorio, el entorno y los cuerpos. Esta es una apuesta política por dos razones. Primero, porque su permanencia en Madrid como trabajadoras cooperativas depende de una agenda intergeneracional de trabajo atada a procesos de autonomía alimentaria que interpelan las iniciativas del tipo de desarrollo que subyace a la ordenación del territorio para los monocultivos y el comercio a gran escala. Segundo, es una apuesta que enfatiza el lugar de la trayectoria laboral y de sus cuerpos en la valoración del empleo en la Sabana Occidente y, por esta razón, va más allá de una lectura del trabajo de las mujeres en la región a partir de cifras de empleabilidad o, simplemente, desde los riesgos y enfermedades profesionales.

El caso de las mujeres de Asoquimad y las delimitantes territoriales aquí enunciadas contrasta las transformaciones de un área periurbana con la posibilidad de autoabastecimiento de alimentos como forma de subsistencia y proyecto de vida para mujeres que transitan fuera de la industria floricultora en busca de una alternativa al trabajo asalariado enajenante de sus cuerpos y su realidad. No obstante, la necesidad de articulación conceptual y metodológica del territorio como categoría analítica no está interesada en hacer una idealización del mismo o de lo popular. De hecho, antes que una nostalgia del territorio como espacio idealizado, las dimensiones territoriales del trabajo que identificamos en la apuesta de las mujeres de Asoquimad por el trabajo cooperativo evidencian que sus reducidas opciones laborales son manifestación de la profunda conexión entre los procesos de reorganización territorial de la Sabana Occidente. En concreto, estas dimensiones brindan elementos para comprender la constitución de un escenario periurbano injusto. Este escenario es el resultado de la intervención territorial de las instituciones locales como respuesta a la decisión de las mujeres de elegir un modo de vida fuera de la producción industrial, pero también de la incidencia de procesos nacionales y globales. El conjunto de factores resaltados dificulta de manera definitiva la posibilidad laboral y política de estas mujeres de producir alimentos como forma de subsistencia. 


\section{Conclusión: lo laboral como relacional}

¿Qué puede decirle la situación de las mujeres de Asoquimad al derecho y a la enseñanza del derecho laboral? En este artículo sugerimos que estas mujeres son actoras del derecho laboral y que las delimitaciones territoriales que su apuesta por la producción de alimentos en pequeña escala y de manera solidaria enfrenta interpelan la manera como el derecho laboral entiende el trabajo. Por ello proponemos que la lectura situada y relacional del trabajo por parte de Asoquimad y la EMM constituye una forma de interpelar a la construcción de lo laboral en el derecho, para que brinde herramientas que permitan no solo identificar las diversas formas contemporáneas de trabajo y sus dimensiones territoriales, sino también la manera como han sido o son inmunizadas al debate democrático en escenarios de profundas transformaciones del suelo y el entorno. La apuesta de Asoquimad exige pensar lo laboral siempre en relación con otras categorías como el territorio, las trayectorias laborales pasadas, los horizontes laborales alternativos, y las historias inscritas y narradas por los cuerpos. Esta apuesta se enfrenta y reta directamente a una mirada reduccionista del trabajo, en general, y del derecho laboral, en particular.

La historia laboral y de relación con el territorio de las mujeres puede nutrir la literatura sobre las estrategias para transformar el uso del suelo desde una perspectiva laboral territorial, y de este modo resistir la producción de escenarios laborales periurbanos injustos. Adicionalmente, el contraste de un área periurbana en rápida transformación con la apuesta de Asoquimad por volver a la tierra permite conectar la pregunta arriba enunciada con debates contemporáneos sobre hacia dónde debe orientarse y qué problemáticas deben responder la academia y la docencia del derecho laboral. Quizás, entonces, sería una conclusión más apropiada preguntar qué podrá decirle la academia del derecho laboral a estas mujeres.

\section{Referencias}

Alcaldía de Madrid (2014). Diagnóstico territorial. Trabajo presentado en Foro Regional de Floricultura: Miradas al Trabajo, el Territorio y su Gente. Madrid, Colombia.

Alcaldía de Mosquera (10 de abril de 2007). Se modifica la reglamentación urbanística para el municipio de Mosquera, departamento de Cundinamarca y se deroga el Decreto 092 de 200o. [Decreto 053 de 2007]. Recuperado de https://mosqueracundinamarca.micolombiadigital.gov.co/sites/mosqueracundinamarca/content/files/oooo33/165o_decreto_053_del_10_de_abril_de_2007.pdf 
Alcaldía de Mosquera (27 de agosto de 2007). Se adopta el Plan Parcial Zona Expansión Industrial Siete Trojes. [Decreto 116 de 2007].

Asociación Ambiente y Sociedad (2017). Conflictos socioambientales en la sabana de Bogotá: el caso del humedal Moyano. Recuperado de https://www.ambienteysociedad.org.co/conflictos-socioambientales-en-la-sabana-de-bogota-caso-humedal-moyano/

Asociación de Zonas Francas de las Américas (2017). Reporte Anual Estadístico, Zonas Francas Colombia. Bogotá: Asociación de Zonas Francas de las Américas. Recuperado de: http:// www.asociacionzonasfrancas.org/user/pages/o4.invierta/o1.directorio/_colombia/Estadisticas\%2oZonas\%2oFrancas\%20Colombia.pdf

Banco Mundial (2009). World Development Report: Reshaping Economic Geography. Washington D. C.: Banco Mundial.

Chadha, Gopal Krishna; Sen, Sucharita; Sharma, Hans Raj (2004). Land Resources. En State of the Indian Farmers. A Millenium Study (Vol. 2) (pp. 320-384). New Delhi: Academic Foundation.

Chant, Silvia; Datu, Kerrwin (2015). Women in Cities: Prosperity or Poverty? A Need for Multi-dimensional and Multi-Spatial Analysis. En The City in Urban Poverty (pp.39-63), editado por Charlotte Lemanski; Colin Marx. Londres: Palgrave Macmillan.

Christodoulidis, Emilios (2018). Labour Constitutionalism in a Genealogical Key. Jurisprudence, 9(2), 413-417. https://doi.org/10.1080/20403313.2018.1451439

Concejo Municipal de Madrid (s.f.). Se revisa y ajusta el Plan Básico de Ordenamiento Territorial del municipio de Madrid. [Acuerdo 017 de 2006]. Recuperado de: http://www. madrid-cundinamarca.gov.co/planes/plan-basico-de-ordenamiento-territorial

Concejo Municipal de Madrid (10 de junio de 200o). Se adopta el Plan Básico de Ordenamiento Territorial del municipio de Madrid. [Acuerdo 024 de 200o]. Recuperado de http:// www.ccb.org.co/La-Camara-CCB/Comite-de-Integracion-Territorial-CIT/Documentos-de-la-Secretaria-tecnica/Biblioteca/Planes-de-Ordenamiento-Territorial

Concejo Municipal de Madrid (10 de junio de 2016). Se adopta el Plan de Desarrollo 20162020 "Buenos vecinos, unidos podemos". [Acuerdo oo5 de 2016]. Recuperado de http:// www.madrid-cundinamarca.gov.co/planes/plan-de-desarrollo-2016-2O2O-buenos-vecinos-unidos-podemos

Congreso de Colombia (30 de diciembre de 2005). Por la cual se modifica un régimen especial para estimular la inversión y se dictan otras disposiciones. [Ley 1004 de 2005]. Diario Oficial, 46.138. Recuperado de http://www.secretariasenado.gov.co/senado/basedoc/ ley_1004_2005.html 
Corte Constitucional (2017). Sentencia C-077/17. [MP Luis Ernesto Vargas Silva]. Recuperado de http://www.corteconstitucional.gov.co/relatoria/2017/C-077-17.htm

Departamento Administrativo Nacional de Estadística (2010). Informe de resultados. Censo de fincas productoras de flores en 28 municipios de la sabana de Bogotá y Cundinamarca 2009. Bogotá: Departamento Administrativo Nacional de Estadística.

Díaz-Caravantes, Rolando Enrique (2012). Balancing Urban and Peri-urban Exchange: Water Geography of Rural Livelihoods in Mexico. Geographical Journal, 178(1), 42-53.

Dukes, Ruth (2014). The Labour Constitution: The Enduring Idea of Labour Law. Oxford: Oxford University Press.

Dukes, Ruth (2018). From the Labour Constitution to an Economic Sociology of Labour Law. Jurisprudence, 9(2), 418-423.

Echeverri, Rafael; Ribero, María Pilar (2002). Nueva ruralidad. Visión del territorio en América Latina y el Caribe. San José, Costa Rica: Instituto Interamericano de Cooperación para la Agricultura.

Flórez, Juliana; Lara-Veloza, Guisella; Veloza, Patricia; Cardozo, Manuel; Espejo, Claudia (2017). Politics of Place at the Women's School of Madrid: Experiences Around Bodies and Territory. En Bodies in Resistance: Gender and Sexual Politics in the Age of Neoliberalism (pp. 25-56), editado por Wendy Harcourt. Londres: Palgrave Macmillan.

Friedmann, John (2016). The Future of Periurban Research. Cities, 53, 163-165.

Halvorsen, Sam (2018). Decolonising Territory: Dialogues with Latin American Knowledges and Grassroots Strategies. Progress in Human Geography, mayo 2018, s.v, s.p. https://doi. org/10.1177/0309132518777623

Harcourt, Wendy (2017). Bodies in Resistance: Gender and Sexual Politics in the Age of Neoliberalism. Londres: Palgrave Macmillan.

Lara-Velosa, Guisella; Veloza-Torres, Patricia; Flórez-Flórez, Juliana (2015). Escuela de Mujeres de Madrid: lugar, corporalidad y trabajos no-capitalistas. Nómadas, 43, 95-111. https://doi. org/10.30578/nomadas.n43a6.

Ministerio del Trabajo de Colombia (2012). Plan local de empleo de Madrid (Cundinamarca), “Empleo en marcha para todos". Bogotá: Ministerio del Trabajo de Colombia.

Pérez, Edelmira (2001). Hacia una nueva visión de lo rural. En ¿Una nueva ruralidad en América Latina? (pp. 17-30), editado por Norma Giarracca. Buenos Aires: CLACSO.

Phillips, Martin (2004). Other Geographies of Gentrification. Progress in Human Geography, 28(1), 5-30. 
Presidencia de Colombia (31 de marzo de 2009). Se establecen condiciones para el cálculo del índice de ocupación en las áreas de desarrollo restringido en suelo rural. [Decreto 1069 de 2009]. Diario Oficial, 47.308. Recuperado de http://www.minambiente.gov.co/images/ normativa/decretos/2009/dec_1069_2009.pdf

Ruoso, Laure-Elise; Plant, Roel (2018). A Politics of Place Framework for Unravelling Peri-urban Conflict: An Example of Peri-urban Sydney, Australia. Journal of Urban Management, $7(2), 57-69$.

Sandoval, María; Robertsdotter, Andrea; Paredes, Myriam (2017). Space, Power and Locality: The Contemporary Use of Territorio in Latin American Geography. Journal of Latin American Geography, 16(1), 43-67.

Secretaría de Desarrollo Económico de Madrid (2016). Estudio previo de mínima cuantía para el servicio de mantenimiento del invernadero y un reservorio de la granja integral demostrativa de la secretaria de desarrollo económico y ambiente del municipio de Madrid, Cundinamarca. Madrid, Colombia: Alcaldía de Madrid.

Sen, Sucharita (2016). Gendered Exclusions in the Work Spaces of Peri-urban Areas in a Neoliberal Environment: Learning from the Experiences of Large Metropolitan Cities in India. Environment and Urbanization ASIA, 7(1), 76-92.

Smit, Jac; Ratta, Annu; Nasr, Joe (2001). Urban Agriculture: Food, Jobs and Sustainable Cities. Nueva York: The Urban Agriculture Network.

Solano Mejía, Augusto (2016) Editorial. Un secreto muy bien guardado. Florycultura colombiana, s. n., 3-4.

Sorensen, Andre (2016). Periurbanization as the Institutionalization of Place: The Case of Japan. Cities, 53, 134-140.

Superintendencia de Sociedades (2016). Desempeño del sector floricultor. Informe. Bogotá: Superintendencia de Sociedades.

Tornaghi, Chiara (2014). Critical Geography of Urban Agriculture. Progress in Human Geography, 38(4), 551-567.

Unidad Administrativa Especial Aeronáutica Civil (6 de diciembre de 2013). Resolución 06815: Plan Maestro del Aeropuerto El Dorado.

Veloza, Patricia; Lara-Veloza, Guisella (2014). De la resignación a la indignación activa: propuesta educativa con mujeres populares de la Asociación Herrera de Madrid, Cundinamarca (Tesis de pregrado). Universidad Pedagógica Nacional, Bogotá, Colombia.

Wekerle, Gerda R.; Classens, Michael (2018). Challenging Property Relations and Access to Land for Urban Food Production. En Urban Gardening as Politics (pp. 105-123), editado por Chiara Tornaghi; Chiara Certomà. Londres: Routledge. 